\title{
NASH Therapy: omega 3 supplementation, vitamin $E$, insulin sensitizers and statin drugs
}

\author{
Stephen Caldwell \\ GI/Hepatology Division, University of Virginia, Charlottesville, Virginia, USA
}

Non-alcoholic steatohepatitis (NASH) is the more aggressive form of non-alcoholic fatty liver disease (NAFLD). NASH can progress to hepatic fibrosis, cirrhosis, portal hypertension and primary liver cancer. Therapy is evolving with a substantial number of trials of promising new agents now in progress. In this article however, we will examine data for several older forms of therapy which have been fairly extensively studied over the years: Polyunsaturated Fatty Acid (PUFA) supplements, vitamin E, insulin sensitizing agents with a focus on pioglitazone and statin agents. Early interest in PUFA derived from their potential benefit in cardio-metabolic disease and the close association of NAFLD/NASH with Metabolic Syndrome. Results have been variable although most studies show reduction of liver fat without other major effects and their effects are influenced by concomitant weight loss and underlying genetic factors. Vitamin $\mathrm{E}$ has had some efficacy in pediatric NASH but questionable efficacy in even mild NASH among adults. Pioglitazone has shown significant histological benefit in a number of trials but concern over side-effects (especially weight gain) have dampened enthusiasm. A newer insulin sensitizer, liraglutide, has also shown promise in a small randomized, controlled trial. Very limited data exists regarding the histological effects of the statins in NASH and these agents appear to be fairly neutral with neither clear cut benefit nor detriment. Their use is best guided by cardiovascular risks rather than liver histology. (Clin Mol Hepatol 2017;23:103-108)

Keywords: Fatty liver; Steatohepatitis; Non-alcoholic steatohepatitis (NASH); Non-alcoholic fatty liver disease (NAFLD); Polyunsaturated Fatty Acid (PUFA)

\section{INTRODUCTION}

Non-alcoholic steatohepatitis (NASH) is a type of non-alcoholic fatty liver disease (NAFLD) that can silently lead to progressive liver damage, hepatic fibrosis, portal hypertension and cirrhosis sometimes further complicated by primary liver cancer. ${ }^{1}$ The condition can be clinically silent for years until it presents suddenly with variceal bleeding or new onset fluid retention such as ascites or hydrothorax. Based on the fundamental pathophysiological mechanisms involved in disease progression which centers on poorly controlled lipid peroxidation or fat rancidification and its relationship to diabetes mellitus and dyslipidemia, several treatment options have undergone clinical study including PUFA supplementation (Poly-Unsaturated Fatty Acids or Omega 3 fatty ac-

\footnotetext{
Abbreviations:

AMP, activated protein kinase-adenosine monophosphate-activated protein kinase; CREBP, Carbohydrate responsive element binding protein; DHA docosahexanoic acid; EPA, eicosapentanoic acid; FXR, farnesoid X receptor; GLA, gamma lenolenic acid; HMG Co, A reductase inhibitor - 3-hydroxy-3-methylglutaryl-coenzyme A reductase inhibitor; HNF-4a, hepatocyte nuclear factor 4 alpha; IU/D, international units per day; LXRa, Liver X receptor alpha; N3, omega 3; N6, omega 6; NAS, NAFLD Activity Score; NASH, Non-alcoholic steatohepatitis; NAFLD, non-alcoholic fatty liver disease; PNPLA3, Patatin-like phospholipase domain-containing protein 3; PPAR, peroxisome proliferator activated receptor; PPAR- $\gamma$, peroxisome-proliferator-activated gamma receptor; PUFA, polyunsaturated fatty acids; RXR, retinoid X receptor; SREBP, Sterol regulatory element-binding proteins; TZDs, thiazolidinediones
}

Corresponding author : Stephen Caldwell

Hepatology Section, University of Virginia, Box 800708, Charlottesville, Virginia 22901, USA

Tel: +1-434-924-2626

Email: shc5c@Virginia.edu 
ids) anti-oxidant therapy with vitamin E (tocopherols), insulin sensitization with anti-diabetic agents such as the 'TZDs' (thiazolidinediones) and, based on the association of NAFLD with dyslipidemia, there has been attention given to anti-cholesterol medications such as the statins. In this discussion, I will highlight some of the work in this area.

\section{OMEGA 3 FATTY ACID (PUFA, OMEGA-3, 'FISH OIL' SUPPLEMENTS)}

PUFA (polyunsaturated fatty acids) are important lipid nutrients which constitute key fatty acid components of the triglyceride/diglyceride and phospholipid membranes of cells and sub-cellular organelle membranes such as the endoplasmic reticulum and the mitochondria. ${ }^{2}$ The fatty acid composition of these phospholipid membranes influences susceptibility to free-radical induced oxidative injury and to activation of inflammatory and anti-inflammatory pathways through their metabolism to structurally related prostaglandins and leukotrienes. These agents also act as ligands for nuclear receptors to stimulate transcription of genes involved in lipid and energy metabolism. ${ }^{3}$

In the most straight forward perspective, PUFA can be seen as consisting of two nutritionally essential 18 carbon fatty acids which are usually ingested as components of ester links of three fatty acids linked to a three carbon glycerol backbone (i.e. triacylglycerides or 'triglycerides'): 1. Linoleic Acid 18:2 $(9,12)$ also known as Omega 6 (N6) fatty acids and 2. Linolenic Acid 18:3 $(9,12,15)$ also known as Omega 3 (N3) fatty acids. The nomenclature and biochemistry of these agents and their downstream metabolites is remarkably complex. 'Unsaturated' refers to the presence of carbo-carbon double bonds (minus a hydrogen bond) with the number of double bonds indicated as 2 or 3 in the formal name and the indication of the position of the 'first' double bond from one terminal of the molecule: hence the terms ' $\mathrm{N} 3$ or N6'. Note that N6 fatty acids have two double bonds and that N3 have three double bonds. They are considered 'essential' because the body cannot synthesize these fatty acids from other precursors. End products of their metabolism include short acting eicosanoids which consist of families of prostaglandins and thromboxanes formed through the activity of cyclooxygenase and lipoxygenase acting on fatty acids cleaved from the membrane bound phospholipids attached to the 3 carbon glycerol backbone.

Through the activities of elongation and desaturation enzymes in the endoplasmic reticulum and the mitochondria, N6 linoleic acid is converted to gamma lenolenic acid (GLA) which is further metabolized (elongated) to arachidonic acid 20:4 (5,8,11,14). N6 PUFA are generally considered pro-inflammatory. Common dietary sources include corn products, safflower seed oil, soy bean oil, and sunflower seeds. In contrast to the N6 fatty acids, N3 Linolenic Acid is, by the same enzymes pathways, further elongated and desaturated to eicosapentanoic acid (EPA) which is further metabolized to Docosahexanoic Acid (DHA). N3 PUFA are generally considered anti-inflammatory because of the properties of their downstream metabolic derivative eicosanoids. Common sources of the N3 fatty acids include fish oil, flax seed, canola, walnuts, and brasil nuts.

Early interest in 'Omega 3' (N3) supplements as possible therapy of NASH can be traced to the close association between the metabolic syndrome (insulin resistance), cardiovascular disease, fatty liver and reports of the cardiovascular benefits of diets rich in omega 3 fatty acids. ${ }^{4-6}$ Interest was further stimulated by the possible role of skeletal muscle phospholipid membrane composition and insulin sensitivity where lower insulin sensitivity was associated with lower skeletal muscle PUFA content thought to represent myocyte phospholipid membrane composition. ${ }^{7}$ Subsequently, lipidomic studies of human fatty liver indicated an association between fatty acid related cellular injury and relatively diminished N3 fatty acids. ${ }^{8}$ In addition, data has accrued which reveals the importance of fatty acids as key nuclear transcription factors which influence the expression of hepatic fat and energy metabolic pathways. ${ }^{9,10}$ These include PPAR receptors (peroxisome proliferator activated receptor), SREBP, CREBP, LXR $\alpha, F X R$, RXR, HNF- $4 \alpha$ all of which influence hepatic fat metabolism.

A number of trials of Omega 3 supplements have been conducted in the treatment of human NASH. Some variation in results is likely explained by nuances of the trials including formulation of omega 3, duration and dose of the supplement, measured target endpoints, control for concomitant exercise and dietary changes, genetic and epigenetic background of the study subjects and factors as subtle as the pattern of supplement ingestion. ${ }^{11,12}$ None of the studies have shown improvement in key prognostic histological features such as fibrosis. However, most but not all trials have shown reduction in hepatic fat content. Using biopsy as the measure of fat content, one study reported no change in steatosis after 12 months of a synthetic EPA supplement up to 2,700 mg/day compared to placebo treated subjects. ${ }^{13}$ In contrast, using MRS to provide a more global measure of liver fat content, the WELCOME trial reported significant hepatic fat reduction after 15-18 months of $4,000 \mathrm{mg} /$ day of a synthetic mixture of EPA and DHA compared 
to placebo (derived from olive oil). ${ }^{14}$ The reduction in liver fat was predictable by changes in erythrocyte N6-N3 phospholipid composition. Similarly, our own study of 12 months of $3,000 \mathrm{mg} /$ day of a fish oil derived mixture of EPA and DHA versus soy bean oil placebo, showed reduced liver fat by the Dixon modified MRI but the effect was significantly less evident in patients who lost even a modest amount of weight during the study period. ${ }^{15}$ As reported by others ${ }^{16}$ the effect was also influenced by the PNPLA3 genotype being greater among individuals with steatosis prone GG allele. Because PUFA are more prone to oxidative injury than saturated fatty acids, it is also notable that none of the trials have observed evidence of increased injury.

Taken together, the effects of omega 3 supplements appear to reduce liver fat overall within a 1-2 year time frame but neither reduce nor exacerbate steatohepatitis. The effect is influenced by both the ability to lose even a modest amount of weight through diet and aerobic activity and by the underlying individual genetic background governing hepatic fat metabolism. Whether or not higher dietary ingestion of N3 fatty acids over a longer term (ie a lifetime) influences the course of fatty liver and NASH remains uncertain but seems plausible. The effect may be mediated by skeletal muscle phospholipid metabolism although we could not detect a beneficial effect on exercise capacity.

\section{VITAMIN E}

Vitamin E constitutes a family of tocopherols and tocotrienols which have been studied over the years in the treatment of NASH based on their well-known anti-oxidant properties and the key role of lipid peroxidation in NASH pathogenesis. ${ }^{17}$ Most studies have used $\alpha$ tocopherol with somewhat variable results. In an early histology based trial, vitamin E (800 IU/d) combined with ursodeoxycholic acid (12-15 mg/kg) with for two years versus single or double placebo controls showed histological improvement in the combination group. ${ }^{18}$ Favorable results were also noted when vitamin E was combined with vitamin C in an early clinical trial. ${ }^{19}$

The most significant effects have been seen in pediatric NASH patients. The TONIC trial randomized 173 pediatric patients to one of three groups for two years of therapy: vitamin $E$ (800 IU/day) $n=58$, metformin $(1,000 \mathrm{mg} /$ day $) \mathrm{n}=57$ and placebo $n=58 .{ }^{20}$ Biopsy was the primary endpoint. Most were obese with evidence of insulin resistance. The mean age was 13 years and $80 \%$ were male and $70 \%$ were Caucasian. The fibrosis stage was mild in all. Using one of the more reliable histological endpoints, the study demonstrated resolution of NASH in follow up biopsy in $28 \%$ in the placebo group, $41 \%$ in the metformin group and, significantly, in $58 \%$ of the vitamin $E$ group.

The results were less impressive in the companion adult trial of vitamin E among adults known as the PIVENS trial which randomized non-diabetic NASH patients to one of three groups: vitamin $E$ (800 IU/day) $n=84$, pioglitazone (30 mg/day) $n=80$, and placebo $n=83$ for two years of therapy. ${ }^{21}$ The fibrosis was stage was mild. While it was one of the largest NASH trials at that time, the study has been criticized for its target population (very mild NASH in the absence of diabetes) and for problems with histological inclusion criteria (variable cellular ballooning) and its primary endpoint of reduction of the histological NAS (NAFLD Activity Score). ${ }^{22}$ A close look at the study results in terms of the more reliable endpoint of NASH resolution showed significantly greater efficacy of pioglitazone (see below) compared to vitamin $E$ which was only marginally better than placebo: pioglitazone: $47 \%(P=0.001)$, vitamin E: $36 \%(P=0.05)$ and placebo: $21 \%$.

Taken together, the data suggest that high dose vitamin $\mathrm{E}$ (800 IU/day) appears to have some benefit in mild NASH among pediatric patients but only a limited effect in adults. Concerns regarding safety with the long term use of the agent ${ }^{23,24}$ and its limited efficacy have dampened enthusiasm although it is notable that no evidence vitamin $\mathrm{E}$ related adverse events were reported during two years of therapy in these trials.

\section{INSULIN SENSITIZING AGENTS}

Because of the close association of fatty liver with insulin resistance and type 2 diabetes, there has been long term interest in the potential benefits of anti-diabetic, insulin sensitizing agents in NASH. Early studies of metformin, a biguanide which alters cellular bioenergetics through AMP-activated protein kinase, ${ }^{25}$ showed favorable effects in experimental rodent models of NASH but subsequent human trials didn't realize a substantial benefit although the lack of weight gain and usually good tolerance (aside from occasional GI side-effects) have sustained interest in the agent among some practitioners. ${ }^{26,27}$ Much greater interest has been focused on the thiazolidinediones or TZD's since the initial report of their use in NASH. ${ }^{28}$ These agents are ligands of the 'gamma' peroxisome-proliferator-activated gamma receptor (PPAR- $\gamma$ ) which is a nuclear transcription receptor activated by fatty acids and expressed in adipocytes, enterocytes and myocytes and influences expression of metabolic pathway components involved in lipid 
and glucose metabolism.

Randomized and controlled trials, including the more recent PIVENS trial noted above, have consistently shown histological benefit with these agents in a sizable minority of treated patients (40-50\% with histological response). ${ }^{21,29,30}$ Similar results have been seen with rosiglitazone although subsequent concerns regarding adverse effects on lipoprotein profiles which raised safety concerns were never resolved. ${ }^{31-33}$ In a more recent study from of $45 \mathrm{mg} /$ day pioglitazone for 18 months of placebo controlled therapy followed by 18 months of open label therapy (101 adults of approximate age 50 years about 1/2 being diabetic and 3/4 of Hispanic descent), consistent results were also seen with resolution of NASH on follow-up biopsy in $51 \%$ of treated patients versus $19 \%$ of placebo treated controls $(P<0.001) .{ }^{34}$

Despite the track record in terms of efficacy with biopsy based evaluation, ${ }^{35,36}$ safety concerns have dampened enthusiasm for use of the TZDs in NASH. ${ }^{37}$ While the purported risk of bladder cancer appears to be a spurious legal miscarriage, ${ }^{38}$ other risks appear to be more genuine..$^{39,40}$ In addition, the effect of increased peripheral fat stores evident as a several kilogram weight gain in many treated patients, while avoidable, has also led to decreased patient acceptance. ${ }^{41}$ Moreover, the lack of studies in more advanced fibrosis stages of NASH where the risk-benefit may be more acceptable significantly limits this group of agents.

The close links between fatty liver (NAFLD), fatty liver with fibrosing steatohepatitis (NASH) and diabetes has led to the study of other, newer anti-diabetic agents recently reported in randomized and controlled trials of NASH. Sitagliptin, a dipeptidyl peptidase-4 (DPP-4) inhibitor, is an orally administered insulin sensitizer which did not show benefit in a controlled trial ${ }^{42}$ while liraglutide, a parenterally administered analogue of glucagon-like peptid-1 (an incretin) did show histological improvement compared to a placebo treated group after approximately one year of therapy in the 'LEAN' trial. ${ }^{43}$ Nine of 23 (39\%) treated patients versus 2 of 22 (9\%) had histological resolution of NASH. Gastrointestinal side-effects were common in both groups but more so in the treated group. Further study seems warranted with longer follow-up and larger study groups.

\section{STATINS}

The use of the HMG Co-A reductase inhibitors or statins remains something of an enigma in terms of their effects in fibrosing steatohepatitis. ${ }^{44}$ Based on one very small prospective trial, ${ }^{45}$ these agents don't overtly benefit or exacerbate NASH although for those with significant vascular risks, the net benefit favors their use when indicated for co-existing vascular risks. ${ }^{46,47}$ In one long term cohort study of serial biopsy in NASH patients with $(n=17)$ and without $(n=51)$ statin therapy, mean fibrosis scores improved in the statin treated group but the percentage of patients with advanced stage fibrosis was higher in the treated group ( $29 \%$ versus $12 \%$ ) at the end $10-15$ years of follow-up. ${ }^{48}$ As with other agents noted above, the patient's PNPLA3 genotype may significantly influence the effect of these medications. ${ }^{49}$ Much more work is needed to clarify their role but presently the best advice is that their use should be governed by conventional risks of vascular disease rather than presence or absence of NASH.

\section{Conflicts of Interest}

The author has no conflicts to disclose.

\section{REFERENCES}

1. Younossi ZM, Koenig AB, Abdelatif D, Fazel Y, Henry L, Wymer M. Global epidemiology of nonalcoholic fatty liver disease: metaanalytic assessment of prevalence, incidence, and outcomes. Hepatology 2016;64:73-84.

2. Champe PC, Harvey RA, Ferrier DR. Biochemistry. 3rd Ed. USA:Lippincott Williams \& Wilkins, 2005:1-534.

3. de Castro GS, Cardoso JF, Calder PC, Jordão AA, Vannucchi H. Fish oil decreases hepatic lipogenic genes in rats fasted and refed on a high fructose diet. Nutrients 2015;7:1644-1656.

4. Lonardo A, Bellentani S, Argo CK, Ballestri S, Byrne CD, Caldwell $\mathrm{SH}$, et al. Epidemiological modifiers of non-alcoholic fatty liver disease: Focus on high-risk groups. Dig Liver Dis 2015;47:997-1006.

5. Burr ML, Fehily AM, Gilbert JF, Rogers S, Holliday RM, Sweetnam PM, et al. Effects of changes in fat, fish, and fibre intakes on death and myocardial reinfarction: diet and reinfarction trial (DART). Lancet 1989;2:757-761.

6. Dietary supplementation with n-3 polyunsaturated fatty acids and vitamin E after myocardial infarction: results of the GISSIPrevenzione trial. Gruppo Italiano per lo Studio della Sopravvivenza nell'Infarto miocardico. Lancet 1999;354:447-455.

7. Borkman M, Storlien LH, Pan DA, Jenkins AB, Chisolm DJ, Campbell LV. The relationship between insulin sensitivity and the fatty acid composition of skeletal muscle. N Engl J Med 1993;328:238-244.

8. Puri P, Baillie RA, Wiest MM, Mirshahi F, Choudhury J, Cheung O, et al. A lipidomic analysis of nonalcoholic fatty liver disease. Hepatology 2007;46:1081-1090 
9. Zheng X, Rivabene R, Cavallari C, Napolitano M, Avella M, Bravo $E$, et al. The effects of chylomicron remnants enriched in $n-3$ or $n-6$ polyunsaturated fatty acids on the transcription of genes regulating their uptake and metabolism by the liver: influence of cellular oxidative state. Free Radic Biol Med 2002;32:1123-1131.

10. Jump DB. N-3 polyunsaturated fatty acid regulation of hepatic gene transcription. Curr Opin Lipidol 2008;19:242-247.

11. Browning LM, Walker CG, Mander AP, West AL, Gambell J, Madden J, et al. Compared with daily, weekly n-3 PUFA intake affects the incorporation of eicosapentaenoic acid and docosahexaenoic acid into platelets and mononuclear cells in humans. J Nutr 2014;144:667672.

12. Sookoian S, Pirola CJ. The genetic epidemiology of nonalcoholic fatty liver disease: toward a personalized medicine. Clin Liver Dis 2012;16:467-485.

13. Sanyal AJ, Abdelmalek MF, Suzuki A, Cummings OW, Chojkier M. No significant effects of ethyl-eicosapentanoic acid on histologic features of nonalcoholic steatohepatitis in a phase 2 trial. Gastroenterology 2014;147:377-384.

14. Scorletti E, Bhatia L, McCormick KG, Clough GF, Nash K, Hodson $L$, et al. Effects of purified eicosapentaenoic and docosahexaenoic acids in nonalcoholic fatty liver disease: results from the Welcome* study. Hepatology 2014;60:1211-1221.

15. Argo CK, Patrie JT, Lackner C, Henry TD, de Lange EE, Weltman AL, et al. Effects of $\mathrm{n}-3$ fish oil on metabolic and histological parameters in NASH: a double-blind, randomized, placebo-controlled trial. J Hepatol 2015;62:190-197.

16. Scorletti E, West AL, Bhatia L, Hoile SP, McCormick KG, Burdge $G C$, et al. Treating liver fat and serum triglyceride levels in NAFLD, effects of PNPLA3 and TM6SF2 genotypes: Results from the WELCOME trial. J Hepatol 2015;63:1476-1483.

17. Brigelius- Flohé $R$, Traber MG. Vitamin E: function and metabolism. FASEB J 1999;13:1145-1155.

18. Dufour JF, Oneta CM, Gonvers JJ, Bihl F, Cerny A, Cereda JM, et al. Randomized placebo-controlled trial of ursodeoxycholic acid with vitamin e in nonalcoholic steatohepatitis. Clin Gastroenterol Hepatol 2006;4:1537-1543.

19. Harrison SA, Torgerson S, Hayashi P, Ward J, Schenker S. Vitamin E and Vitamin $C$ treatment improves fibrosis in patients with nonalcoholic steatohepatitis. Am J Gastroenterol 2003;98:2485-2490.

20. Lavine JE, Schwimmer JB, Van Natta ML, Molleston JP, Murray KF, Rosenthal $P$, et al. Effect of vitamin $E$ or metformin for treatment of nonalcoholic fatty liver disease in children and adolescents: the TONIC randomized controlled trial. JAMA 2011;305:1659-1668.

21. Sanyal AJ, Chalasani N, Kowdley KV, McCullough A, Diehl AM, Bass $N M$, et al. Pioglitazone, vitamin $E$, or placebo for nonalcoholic steatohepatitis. N Engl J Med 2010;362:1675-1685.

22. Argo CK, Ikura Y, Lackner C, Caldwell SH. The fat droplet in hepato- cellular ballooning and implications for scoring nonalcoholic steatohepatitis therapeutic response. Hepatology 2016;63:1056-1057.

23. Miller ER, Pastor-Barriuso R, Dalal D, Riemersma RA, Appel LJ, Guallar E. Meta-Analysis: high-dosage Vitamin E supplementation may increase all-cause mortality. Ann Intern Med 2005;142:37-46.

24. Brown BG, Crowley J. Is there any hope for vitamin E? JAMA 2005;293:1387-1390.

25. Zhou G, Myers R, Li Y, Chen Y, Shen X, Fenyk-Melody J, et al. Role of AMP-activated protein kinase in mechanism of metformin action. J Clin Invest 2001;108:1167-1174.

26. Bugianesi E, Gentilcore E, Manini R, Natale S, Vanni E, Villanova N, et al. A randomized controlled trial of metformin versus vitamin $E$ or prescriptive diet in nonalcoholic fatty liver disease. Am J Gastroenterol 2005:100:1082-1090.

27. Uygun A, Kadayifci A, Isik AT, Ozgurtas T, Deveci S, Tuzun A, et al. Metformin in the treatment of patients with non-alcoholic steatohepatitis. Aliment Pharmacol Ther 2004;19:537-544.

28. Caldwell SH, Hespenheide EE, Redick JA, lezzoni JC, Battle EH, Sheppard BL. A pilot study of a thiazolidinedione, troglitazone, in nonalcoholic steatohepatitis. Am J Gastroenterol 2001;96:519-525.

29. Belfort R, Harrison SA, Brown K, Darland C, Finch J, Hardies J, et al. A placebo-controlled trial of pioglitazone in subjects with nonalcoholic steatohepatitis. N Engl J Med 2006;355:2297-2307.

30. Aithal GP, Thomas JA, Kaye PV, Lawson A, Ryder SD, Spendlove I, et al. Randomized, placebo-controlled trial of pioglitazone in nondiabetic subjects with nonalcoholic steatohepatitis. Gastroenterology 2008;135:1176-1184

31. Ratziu V, Giral P, Jacqueminet S, Charlotte F, Hartemann-Heurtier A, Serfaty L, et al. Rosiglitazone for nonalcoholic steatohepatitis: one-year results of the randomized placebo-controlled Fatty Liver Improvement with Rosiglitazone Therapy (FLIRT) Trial. Gastroenterology 2008;135:100-110.

32. Ratziu V, Charlotte F, Bernhardt C, Giral P, Halbron M, Lenaour G, et al. Long-term efficacy of rosiglitazone in nonalcoholic steatohepatitis: results of the fatty liver improvement by rosiglitazone therapy (FLIRT 2) extension trial. Hepatology 2010;51:445-453.

33. Ratziu V, Caldwell S, Neuschwander-Tetri BA. Therapeutic trials in nonalcoholic steatohepatitis: insulin sensitizers and related methodological issues. Hepatology 2010;52:2206-2215.

34. Cusi K, Orsak B, Bril F, Lomonaco R, Hecht J, Ortiz-Lopez C, et al. Long-term pioglitazone treatment for patients with nonalcoholic steatohepatitis and prediabetes or type 2 diabetes mellitus: a randomized trial. Ann Intern Med 2016;165:305-315.

35. Mahady SE, Webster AC, Walker S, Sanyal A, George J. The role of thiazolidinediones in non-alcoholic steatohepatitis - a systematic review and meta analysis. J Hepatol 2011;55:1383-1390.

36. Boettcher E, Csako G, Pucino F, Wesley R, Loomba R. Meta-analysis: pioglitazone improves liver histology and fibrosis in patients with non- 
alcoholic steatohepatitis. Aliment Pharmacol Ther 2012;35:66-75.

37. Nesto RW, Bell D, Bonow RO, Fonseca V, Grundy SM, Horton ES, et al. Thiazolidinedione use, fluid retention, and congestive heart failure: Consensus statement from the American Heart Association and the American Diabetes Association. Circulation 2003;108:29412948.

38. Davidson MB. Pioglitazone (Actos) and bladder cancer: Legal system triumphs over the evidence. J Diabetes Complications 2016;30:981985.

39. Nissen SE, Wolski K. Effect of rosiglitazone on the risk of myocardial infarction and death from cardiovascular causes. N Engl J Med 2007;356:2457-2471.

40. Lincoff AM, Wolski K, Nicholls SJ, Nissen SE. Pioglitazone and risk of cardiovascular events in patients with type 2 diabetes mellitus: a meta-analysis of randomized trials. JAMA 2007;298:1180-1188.

41. Argo CK, lezzoni JC, Al-Osaimi AM, Caldwell SH. Thiazolidinediones for the treatment in NASH: sustained benefit after drug discontinuation? J Clin Gastroenterol 2009:43:565-568.

42. Cui J, Philo L, Nguyen P, Hofflich $H$, Hernandez C, Bettencourt R, et al. Sitagliptin vs. placebo for non-alcoholic fatty liver disease: $A$ randomized controlled trial. J Hepatol 2016;65:369-376.

43. Armstrong MJ, Gaunt P, Aithal GP, Barton D, Hull D, Parker R, et al.
Liraglutide safety and efficacy in patients with non-alcoholic steatohepatitis (LEAN): a multicentre, double-blind, randomised, placebocontrolled phase 2 study. Lancet 2016;387:679-690.

44. Argo CK, Loria P, Caldwell SH, Lonardo A. Statins in liver disease: a molehill, an iceberg, or neither? Hepatology 2008;48:662-669.

45. Nelson A, Torres DM, Morgan AE, Fincke C, Harrison SA. A pilot study using simvastatin in the treatment of nonalcoholic steatohepatitis: A randomized placebo-controlled trial. J Clin Gastroenterol 2009;43:990-904.

46. Vuppalanchi R, Chalasani N. Statins for hyperlipidemia in patients with chronic liver disease: are they safe? Clin Gastroenterol Hepatol 2006;4:838-839.

47. Eslami L, Merat S, Malekzadeh R, Nasseri-Moghaddam S, Aramin H. Statins for non-alcoholic fatty liver disease and non-alcoholic steatohepatitis. Cochrane Database Syst Rev 2013;(12):CD008623.

48. Ekstedt M, Franzén LE, Mathiesen UL, Holmqvist M, Bodemar G, Kechagias $S$. Statins in non-alcoholic fatty liver disease and chronically elevated liver enzymes: a histopathological follow-up study. J Hepatol 2007;47:135-141.

49. Dongiovanni P, Petta S, Mannisto V, Mancina RM, Pipitone R, Karja V, et al. Statin use and non-alcoholic steatohepatitis in at risk individuals. J Hepatol 2015;63:705-712. 